\title{
Higher-Order Amplitude Squeezing in Six-Wave Mixing Process
}

\author{
Sunil Rani, ${ }^{1}$ Jawahar Lal, ${ }^{2}$ and Nafa Singh ${ }^{3}$ \\ ${ }^{1}$ Department of Applied Physics, Shri Krishan Institute of Engineering \& Technology, Kurukshetra 136118, India \\ ${ }^{2}$ Department of Physics, Markanda National College, Shahbad, Kurukshetra 136118, India \\ ${ }^{3}$ Department of Physics, Kurukshetra University, Kurukshetra 136119, India
}

Correspondence should be addressed to Sunil Rani, sunil.lather@gmail.com

Received 28 April 2010; Accepted 11 April 2011

Academic Editor: A. Cartaxo

Copyright () 2011 Sunil Rani et al. This is an open access article distributed under the Creative Commons Attribution License, which permits unrestricted use, distribution, and reproduction in any medium, provided the original work is properly cited.

\begin{abstract}
We investigate theoretically the generation of squeezed states in spontaneous and stimulated six-wave mixing process quantum mechanically. It has been found that squeezing occurs in field amplitude, amplitude-squared, amplitude-cubed, and fourth power of field amplitude of fundamental mode in the process. It is found to be dependent on coupling parameter "g" (characteristics of higher-order susceptibility tensor) and phase values of the field amplitude under short-time approximation. Six-wave mixing is a process which involves absorption of three pump photons and emission of two probe photons of the same frequency and a signal photon of different frequency. It is shown that squeezing is greater in a stimulated interaction than the corresponding squeezing in spontaneous process. The degree of squeezing depends upon the photon number in first and higher orders of field amplitude. We study the statistical behaviour of quantum field in the fundamental mode and found it to be sub-Poissonian in nature. The signalto-noise ratio has been studied in different orders. It is found that signal-to-noise ratio is higher in lower orders. This study when supplemented with experimental observations offers possibility of improving performance of many optical devices and optical communication networks.
\end{abstract}

\section{Introduction}

Over the past three decades, particular attention has been focused on theoretical investigations and experimental observations in generation of squeezed light, for improving the performance of many optical devices and optical communication networks. The concept of squeezed light is concerned with reduction of quantum fluctuations in one of the quadrature, at the expense of increased fluctuations in the other quadrature. In general, the two important nonclassical effects, squeezing and antibunching (or SubPoissonian photon statistics), are not interrelated; that is, some states exist that exhibit the first but not the second and vice versa. However, squeezing can be detected using simple photon counting in higher-order sub-Poissonian statistics.

A lot of work has appeared in the literature on the theoretical and experimental investigations on generation of squeezed states of electromagnetic field. Mandel [1] found squeezed state of the second harmonic when a beam of light propagates through a nonlinear crystal. Later, Hillery [2] defined amplitude-squared squeezing and showed that amplitude-squared squeezed states can be of use in reducing noise in the output of certain nonlinear optical devices. Hong and Mandel [3, 4] introduced the notion of $N$ th-order squeezing as a generalization of the secondorder squeezing. Zhan [5] proposed the generation of amplitude-cubed squeezing in the fundamental mode in second and third harmonic generation. Jawahar and Jaiswal [6] extended the results obtained by Zhan for amplitudecubed squeezing in the fundamental mode during second and third harmonic generations to $k$ th order. The significant experimental observations include gravity wave detection [7-10], in optical communication [11], in nanodisplacement measurement [12], and in optical storage [13], and interferometer enhancement $[14,15]$. The experimental detections and applications confirm the importance of the theoretical investigations into various optical processes such as four- and six-wave mixing [16-20], eight-wave mixing [21], higher-order harmonic generation [22-25], parametric amplification [26], Raman [27] and hyper-Raman processes [28], and so forth. Higher-order sub-Poissonian statistics have been studied by a number of authors such as those in 
[29-31]. The conversion of higher-order squeezed light into nonclassical light with high sub-Poissonian statistics and its experimental detection has been discussed in [31-33].

Recently, Giri and Gupta [19] have investigated the squeezing effects in six-wave mixing process. In this paper, we propose a different model for the same interaction process. Also, this paper shows one of the distinguished examples of nonlinear processes when light exhibits both squeezing and sub-Poissonian photon statistics at the same time. Squeezing in field amplitude, amplitude-squared, amplitude-cubed, and in fourth-order amplitude has been studied in fundamental mode for the proposed model. The photon statistics and dependence of squeezing on photon number have also been investigated.

\section{Definition of Squeezing and Higher-Order Squeezing}

Squeezing is a purely quantum mechanical phenomenon which cannot be explained on the basis of classical physics. The coherent states do not exhibit nonclassical effects, but a superposition of coherent states can exhibit normal squeezing, higher-order squeezing, and sub-Poissonian photon statistics. A coherent state changes to a superposition of coherent states when it interacts with a non linear medium. Squeezed states of an electromagnetic field are the states with reduced noise below the vacuum limit in one of the canonical conjugate quadratures. Normal squeezing is defined in terms of the operators

$$
X_{1}=\frac{1}{2}\left(A+A^{\dagger}\right), \quad X_{2}=\frac{1}{2 i}\left(A-A^{\dagger}\right),
$$

where $X_{1}$ and $X_{2}$ are the real and imaginary parts of the field amplitude, respectively. $A$ and $A^{\dagger}$ are slowly varying operators defined by

$$
A=a e^{i \omega t}, \quad A^{\dagger}=a^{\dagger} e^{-i \omega t} .
$$

The operators $X_{1}$ and $X_{2}$ obey the commutation relation

$$
\left[X_{1}, X_{2}\right]=\frac{i}{2}
$$

which leads to the uncertainty relation $(\hbar=1)$

$$
\Delta X_{1} \Delta X_{2} \geq \frac{1}{4}
$$

A quantum state is squeezed in $X_{i}$ variable if

$$
\Delta X_{i}<\frac{1}{2} \text { for } i=1 \text { or } 2 .
$$

Amplitude-squared squeezing is defined in terms of operators $Y_{1}$ and $Y_{2}$ as

$$
Y_{1}=\frac{1}{2}\left[A^{2}+A^{\dagger 2}\right], \quad Y_{2}=\frac{1}{2 i}\left[A^{2}-A^{\dagger 2}\right] .
$$

The operators $Y_{1}$ and $Y_{2}$ obey the commutation relation $\left[Y_{1}, Y_{2}\right]=i(2 N+1)$, where $N$ is the usual number operator which leads to the uncertainty relation

$$
\Delta Y_{1} \Delta Y_{2} \geq\left\langle\left(N+\frac{1}{2}\right)\right\rangle \text {. }
$$

Amplitude-squared squeezing is said to exist in $Y_{i}$ variable if

$$
\left(\Delta Y_{i}\right)^{2}<\left\langle\left(N+\frac{1}{2}\right)\right\rangle \quad \text { for } i=1 \text { or } 2 .
$$

Amplitude-cubed squeezing is defined in terms of the operators

$$
Z_{1}=\frac{1}{2}\left(A^{3}+A^{t^{3}}\right), \quad Z_{2}=\frac{1}{2 i}\left(A^{3}-A^{t^{3}}\right) .
$$

The operators $Z_{1}$ and $Z_{2}$ obey the commutation relation

$$
\left[Z_{1}, Z_{2}\right]=\frac{i}{2}\left(9 N^{2}+9 N+6\right) .
$$

Relation (10) leads to the uncertainty relation

$$
\Delta Z_{1} \Delta Z_{2} \geq \frac{1}{4}\left(9 N^{2}+9 N+6\right) .
$$

Amplitude-cubed squeezing exists when

$$
\left(\Delta Z_{i}\right)^{2}<\frac{1}{4}\left\langle\left(9 N^{2}+9 N+6\right)\right\rangle \quad \text { for } i=1 \text { or } 2 .
$$

Real and imaginary parts of fourth-order amplitude are given as

$$
F_{1}=\frac{1}{2}\left(A^{4}+A^{\dagger 4}\right), \quad F_{2}=\frac{1}{2 i}\left(A^{4}-A^{\dagger 4}\right) .
$$

The operators $F_{1}$ and $F_{2}$ obey the commutation relation

$$
\left[F_{1}, F_{2}\right]=\frac{i}{2}\left(16 N^{3}+24 N^{2}+56 N+24\right)
$$

and satisfy the uncertainty relation $(\hbar=1)$

$$
\Delta F_{1} \Delta F_{2} \geq \frac{1}{4}\left\langle\left(16 N^{3}+24 N^{2}+56 N+24\right)\right\rangle .
$$

Fourth-order squeezing exists when

$$
\left(\Delta F_{i}\right)^{2}<\frac{1}{4}\left\langle\left(16 N^{3}+24 N^{2}+56 N+24\right)\right\rangle \quad \text { for } i=1 \text { or } 2 .
$$

\section{Squeezing in Fundamental Mode in Six-Wave Mixing Process}

The model considers the process involving absorption of three pump photons of frequency $\omega_{1}$ each, going from state $|1\rangle$ to state $|2\rangle$ and emission of two probe photons from state $|2\rangle$ to state $|3\rangle$ with frequency $\omega_{2}$ each. The atomic system returns to its original state by emitting one signal photon of frequency $\omega_{3}$ from state $|3\rangle$ to $|1\rangle$. The process is shown in Figure 1.

The Hamiltonian for this process is as follows $(\hbar=1)$

$$
H=\omega_{1} a^{\dagger} a+\omega_{2} b^{\dagger} b+\omega_{3} c^{\dagger} c+g\left(a^{3} b^{\dagger 2} c^{\dagger}+a^{\dagger 3} b^{2} c\right),
$$

in which $g$ is a coupling constant. $A=a e^{i \omega_{1} t}, B=b e^{i \omega_{2} t}$, and $C=c e^{i \omega_{3} t}$, respectively, are the slowly varying operators for the three modes at $\omega_{1}, \omega_{2}$, and $\omega_{3} . a\left(a^{\dagger}\right), b\left(b^{\dagger}\right), c\left(c^{\dagger}\right)$ are the 


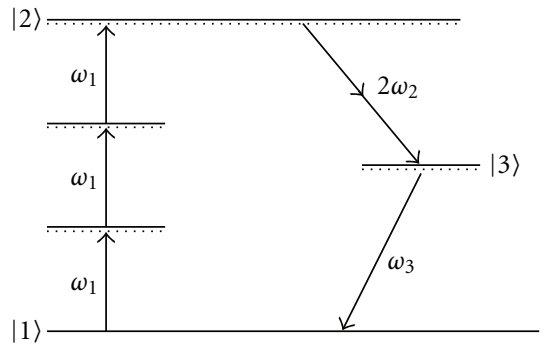

Figure 1: Six-wave interaction model.

usual annihilation (creation) operators associated with the relation $3 \omega_{1}=2 \omega_{2}+\omega_{3}$.

The Heisenberg equation of motion for mode $A$ is

$$
\dot{A}=\frac{\partial A}{\partial t}+i[H, A]
$$

Using (17) in (18), we obtain

$$
\dot{A}=-3 i g A^{\dagger 2} B^{2} C \text {. }
$$

Similarly, we obtained the relations for $\dot{B}$ and $\dot{C}$ as

$$
\begin{gathered}
\dot{B}=-2 i g A^{3} B^{\dagger} C^{\dagger}, \\
\dot{C}=-i g A^{3} B^{\dagger 2} .
\end{gathered}
$$

Expanding $A(t)$ using Taylor's series expansion by assuming the short-time interaction of waves with the medium and retaining the terms up to $|g t|^{2}$, we obtain

$$
\begin{aligned}
A(t)=A- & 3 i g t A^{\dagger 2} B^{2} C+\frac{3}{2} g^{2} t^{2} \\
\times & 6 A^{\dagger} A^{2} B^{\dagger 2} B^{2} C^{\dagger} C+6 A B^{\dagger 2} B^{2} C^{\dagger} C \\
& -4 A^{\dagger 2} A^{3} B^{\dagger} B C^{\dagger} C \\
& -2 A^{\dagger 2} A^{3} C^{\dagger} C-A^{\dagger 2} A^{3} B^{\dagger 2} B^{2} C^{\dagger} C \\
& \left.-4 A^{\dagger 2} A^{3} B^{\dagger} B C^{\dagger} C-2 A^{\dagger 2} A^{3}\right) .
\end{aligned}
$$

The real quadrature component for squeezing of field amplitude in fundamental mode $A$ is given as

$$
X_{1 A}(t)=\frac{1}{2}\left[A(t)+A^{\dagger}(t)\right] .
$$

For spontaneous interaction, we consider the quantum state as a product of coherent state for the fundamental mode $A$ and the vacuum state for the modes $B$ and $C$, that is,

$$
|\psi\rangle=|\alpha\rangle_{A}|0\rangle_{B}|0\rangle_{C},
$$

where $\alpha$ is the complex field amplitude of the fundamental mode. Using (21)-(23), we obtain the expectation values as

$$
\begin{aligned}
& \left\langle\psi\left|X_{1 A}^{2}(t)\right| \psi\right\rangle \\
& =\frac{1}{4}\left[\alpha^{2}+\alpha^{* 2}+2|\alpha|^{2}+1\right. \\
& \left.\quad-6 g^{2} t^{2}\left(\alpha^{2}|\alpha|^{4}+\alpha^{2}|\alpha|^{2}+\alpha^{* 2}|\alpha|^{4}+\alpha^{* 2}|\alpha|^{2}+2|\alpha|^{6}\right)\right],
\end{aligned}
$$

$$
\begin{aligned}
& \left\langle\psi\left|X_{1 A}(t)\right| \psi\right\rangle^{2} \\
& =\frac{1}{4}\left[\alpha^{2}+\alpha^{* 2}+2|\alpha|^{2}-6 g^{2} t^{2}\left(\alpha^{2}|\alpha|^{4}+\alpha^{* 2}|\alpha|^{4}+2|\alpha|^{6}\right)\right] .
\end{aligned}
$$

Therefore,

$$
\begin{aligned}
{\left[\Delta X_{1 A}(t)\right]^{2} } & =\left\langle X_{1 A}^{2}(t)\right\rangle-\left\langle X_{1 A}(t)\right\rangle^{2} \\
& =\frac{1}{4}\left[1-6 g^{2} t^{2}\left(\alpha^{2}|\alpha|^{2}+\alpha^{* 2}|\alpha|^{2}\right)\right], \\
{\left[\Delta X_{1 A}(t)\right]^{2}-\frac{1}{4} } & =-3 g^{2} t^{2}|\alpha|^{4} \cos 2 \theta,
\end{aligned}
$$

where $\theta$ is the phase angle, with $\alpha=|\alpha| e^{i \theta}$ and $\alpha^{*}=|\alpha| e^{-i \theta}$.

The right-hand side of the expression (27) is negative, indicating that squeezing will occur in the first-order amplitude in the fundamental mode in six-wave mixing process for which $\cos 2 \theta>0$ for spontaneous interaction.

In parallel to the spontaneous interaction, the stimulated emission is caused due to the coupling of the atom to the other states of the field. Therefore, the study of squeezing in stimulated interaction in six-wave mixing process requires initial quantum state as a product of coherent states for modes 1,2 and vacuum state for 3 , that is,

$$
|\psi\rangle=|\alpha\rangle_{A}|\beta\rangle_{B}|0\rangle_{C}
$$

Retaining the terms up to $g^{2} t^{2}$, we obtain

$$
\begin{aligned}
& \left\langle\psi\left|X_{1 A}(t)\right| \psi\right\rangle^{2} \\
& =\frac{1}{4}\left[\alpha^{2}+\alpha^{* 2}+2|\alpha|^{2}-3 g^{2} t^{2}\left(\alpha^{2}|\alpha|^{4}+\alpha^{* 2}|\alpha|^{4}+2|\alpha|^{6}\right)\right. \\
& \left.\quad \quad \times\left(|\beta|^{4}+4|\beta|^{2}+2\right)\right], \\
& \left\langle\psi\left|X_{1 A}^{2}(t)\right| \psi\right\rangle \\
& =\frac{1}{4}\left[\alpha^{2}+\alpha^{* 2}+2|\alpha|^{2}+1-3 g^{2} t^{2}\right. \\
& \quad \times\left(\alpha^{2}|\alpha|^{4}+\alpha^{2}|\alpha|^{2}+\alpha^{* 2}|\alpha|^{4}+\alpha^{* 2}|\alpha|^{2}+2|\alpha|^{6}\right) \\
& \left.\quad \times\left(|\beta|^{4}+4|\beta|^{2}+2\right)\right] .
\end{aligned}
$$

Therefore,

$$
\left[\Delta X_{1 A}(t)\right]^{2}-\frac{1}{4}=-\frac{3}{2} g^{2} t^{2}|\alpha|^{4}\left(|\beta|^{4}+4|\beta|^{2}+2\right) \cos 2 \theta,
$$


which is negative, indicating that squeezing will occur for those values of $\theta$ for which $\cos 2 \theta>0$, in the fundamental mode in stimulated interaction under short-time approximation. The effect of the stimulated interaction is represented by the factor $\left(|\beta|^{4}+4|\beta|^{2}+2\right)$.

Using (21) and (23), the second-order amplitude is expressed as

$$
\begin{aligned}
A^{2}(t)= & A^{2}-\operatorname{6igt}\left(A^{\dagger 2} A+A^{\dagger}\right) B^{2} C-3 g^{2} t^{2} \\
& \times\left(A^{\dagger 2} A^{4}+A^{\dagger} A^{3}\right)\left(B^{\dagger 2} B^{2}+4 B^{\dagger} B+2\right) .
\end{aligned}
$$

For second-order squeezing, the real quadrature component for the fundamental mode is expressed as

$$
Y_{1 A}(t)=\frac{1}{2}\left[A^{2}(t)+A^{\dagger 2}(t)\right]
$$

Using (23) and (31) in (32), we get the expectation values in spontaneous six-wave mixing process as

$$
\begin{aligned}
& \left\langle\psi\left|Y_{1 A}(t)\right| \psi\right\rangle^{2} \\
& =\frac{1}{4}\left[\alpha^{4}+\alpha^{* 4}+2|\alpha|^{4}-12 g^{2} t^{2}\right. \\
& \quad \times\left(\alpha^{4}|\alpha|^{4}+\alpha^{4}|\alpha|^{2}+\alpha^{* 4}|\alpha|^{4}+\alpha^{* 4}|\alpha|^{2}+2|\alpha|^{8}\right. \\
& \left.\left.\quad+2|\alpha|^{6}\right)\right], \\
& \begin{aligned}
\left\langle\psi\left|Y_{1 A}^{2}(t)\right| \psi\right\rangle \\
=\frac{1}{4}\left[\alpha^{4}+\alpha^{* 4}+2|\alpha|^{4}+4|\alpha|^{2}+2-12 g^{2} t^{2}\right. \\
\quad \times\left(\alpha^{4}|\alpha|^{4}+3 \alpha^{4}|\alpha|^{2}+2 \alpha^{4}+\alpha^{* 4}|\alpha|^{4}+3 \alpha^{* 4}|\alpha|^{2}\right. \\
\left.\left.\quad+2 \alpha^{* 4}+2|\alpha|^{8}+4|\alpha|^{6}\right)\right] .
\end{aligned}
\end{aligned}
$$

Therefore,

$$
\begin{aligned}
& {\left[\Delta Y_{1 A}(t)\right]^{2}} \\
& =\left\langle Y_{1 A}^{2}(t)\right\rangle-\left\langle Y_{1 A}(t)\right\rangle^{2} \\
& =\frac{1}{4}\left[4|\alpha|^{2}+2-24 g^{2} t^{2}\left(\alpha^{4}|\alpha|^{2}+\alpha^{4}+\alpha^{* 4}|\alpha|^{2}+\alpha^{* 4}+|\alpha|^{6}\right)\right] .
\end{aligned}
$$

The number of photons in mode $A$ may be expressed as

$$
\begin{aligned}
N_{1 A}(t)= & A^{\dagger}(t) A(t) \\
= & A^{\dagger} A+3 \operatorname{igt}\left(A^{3} B^{\dagger 2} C^{\dagger}-A^{\dagger 3} B^{2} C\right) \\
& -3 g^{2} t^{2} A^{\dagger 3} A^{3}\left(B^{\dagger 2} B^{2}+4 B^{\dagger} B+2\right) \\
& +9 g^{2} t^{2}\left(A^{\dagger 2} A^{2}+4 A^{\dagger} A+2\right) B^{\dagger 4} B^{4} C^{\dagger} C .
\end{aligned}
$$

Thus, using condition (23), we get

$$
\left\langle N_{1 A}(t)+\frac{1}{2}\right\rangle=\left[|\alpha|^{2}+\frac{1}{2}-6 g^{2} t^{2}|\alpha|^{6}\right] .
$$

Subtracting (37) from (35), we get

$$
\left[\Delta Y_{1 A}(t)\right]^{2}-\left\langle N_{1 A}(t)+\frac{1}{2}\right\rangle=-12 g^{2} t^{2}\left(|\alpha|^{6}+|\alpha|^{4}\right) \cos 4 \theta .
$$

Using initial condition (28), we obtain squeezing for the stimulated process as

$$
\begin{aligned}
{\left[\Delta Y_{1 A}(t)\right]^{2}-\left\langle N_{1 A}(t)+\frac{1}{2}\right\rangle=} & -6 g^{2} t^{2}\left(|\alpha|^{6}+|\alpha|^{4}\right) \\
& \times\left(|\beta|^{4}+4|\beta|^{2}+2\right) \cos 4 \theta .
\end{aligned}
$$

The right-hand sides of (38) and (39) are negative for all values of $\theta$ for which $\cos 4 \theta>0$ and thus shows the existence of squeezing in the second order of the field amplitude in spontaneous and stimulated interaction under short-time approximation.

Using (21), cubed-amplitude is expressed as

$$
\begin{aligned}
A^{3}(t)= & A^{3}-3 \operatorname{igt}\left(3 A^{\dagger 2} A^{2}+6 A^{\dagger} A+2\right) B^{2} C \\
& -18 g^{2} t^{2}\left(A^{\dagger 4} A+3 A^{\dagger 3}\right) B^{4} C^{2}-\frac{3}{2} g^{2} t^{2} \\
& \times\left(3 A^{\dagger 2} A^{5}+6 A^{\dagger} A^{4}+2 A^{3}\right)\left(B^{\dagger 2} B^{2}+4 B^{\dagger} B+2\right),
\end{aligned}
$$

and the real quadrature component for third-order squeezing in the fundamental mode is expressed as

$$
\begin{aligned}
& Z_{1 A}(t) \\
& =\frac{1}{2}\left[A^{3}(t)+A^{\dagger 3}(t)\right] \\
& =\frac{1}{2}\left[A^{3}+A^{\dagger 3}-3 \operatorname{igt}\left(A^{\dagger 2} A^{2}+6 A^{\dagger} A+2\right) B^{2} C\right. \\
& +3 i g t\left(A^{\dagger 2} A^{2}+6 A^{\dagger} A+2\right) B^{\dagger 2} C^{\dagger} \\
& -18 g^{2} t^{2}\left(A^{\dagger 4} A+3 A^{\dagger 3}\right) B^{4} C^{2} \\
& -18 g^{2} t^{2}\left(A^{\dagger} A^{4}+3 A^{3}\right) B^{\dagger 4} C^{\dagger 2}-\frac{3}{2} g^{2} t^{2} \\
& \left.\times\left(3 A^{\dagger 5} A^{2}+6 A^{\dagger 4} A+2 A^{\dagger 3}+3 A^{\dagger 2} A^{5}+6 A^{\dagger} A^{4}+2 A^{3}\right)\right] .
\end{aligned}
$$

Using (23) and (41), we get the expectation values for spontaneous interaction as

$$
\begin{aligned}
&\left\langle\psi\left|Z_{1 A}(t)\right| \psi\right\rangle^{2} \\
&=\frac{1}{4}\left[\alpha^{6}+\alpha^{* 6}+2|\alpha|^{6}-6 g^{2} t^{2}\right. \\
&\left.\quad \times\left(3|\alpha|^{4}+6|\alpha|^{2}+2\right)\left(\alpha^{6}+\alpha^{* 6}+2|\alpha|^{6}\right)\right],
\end{aligned}
$$




$$
\begin{aligned}
\left\langle\psi\left|Z_{1 A}^{2}(t)\right| \psi\right\rangle & \\
=\frac{1}{4}[ & \alpha^{6}+\alpha^{* 6}+2|\alpha|^{6}+9|\alpha|^{4}+18|\alpha|^{2}+6 \\
& \quad-6 g^{2} t^{2}\left(3|\alpha|^{4}+15|\alpha|^{2}+20\right)\left(\alpha^{6}+\alpha^{* 6}\right) \\
& \left.+6|\alpha|^{10}+30|\alpha|^{8}+40|\alpha|^{6}\right] .
\end{aligned}
$$

Subtracting (42) from (43), we get

$$
\begin{aligned}
{\left[\Delta Z_{1 A}(t)\right]^{2}=\frac{1}{4}[} & {\left[9|\alpha|^{4}+18|\alpha|^{2}+6-54 g^{2} t^{2}\right.} \\
& \left.\times\left(|\alpha|^{2}+2\right)\left(\alpha^{6}+\alpha^{* 6}\right)+2|\alpha|^{8}+4|\alpha|^{6}\right] .
\end{aligned}
$$

Using (23) and (36), we have

$$
\begin{aligned}
& \frac{1}{4}\left\langle 9 N_{1 A}^{2}(t)+9 N_{1 A}(t)+6\right\rangle \\
& \quad=\frac{1}{4}\left[9|\alpha|^{4}+18|\alpha|^{2}+6-108 g^{2} t^{2}\left(|\alpha|^{8}+2|\alpha|^{6}\right)\right] .
\end{aligned}
$$

Subtracting (45) from (44), we get

$$
\begin{gathered}
{\left[\Delta Z_{1 A}(t)\right]^{2}-\frac{1}{4}\left\langle 9 N_{1 A}^{2}(t)+9 N_{1 A}(t)+6\right\rangle} \\
=-27 g^{2} t^{2}\left(|\alpha|^{8}+2|\alpha|^{6}\right) \cos 6 \theta .
\end{gathered}
$$

Using (28), we obtain the stimulated process as

$$
\begin{aligned}
& {\left[\Delta Z_{1 A}(t)\right]^{2}-\frac{1}{4}\left\langle 9 N_{1 A}^{2}(t)+9 N_{1 A}(t)+6\right\rangle} \\
& \quad=-\frac{27}{2} g^{2} t^{2}\left(|\alpha|^{8}+2|\alpha|^{6}\right)\left(|\beta|^{4}+4|\beta|^{2}+2\right) \cos 6 \theta .
\end{aligned}
$$

The right-hand sides of (46) and (47) are negative, for all values of $\theta$ for which $\cos 6 \theta>0$, indicating the existence of squeezing in cubed amplitude in the fundamental mode in the spontaneous and stimulated processes.

For fourth-order squeezing, amplitude is expressed as

$$
\begin{aligned}
A^{4}(t)= & A^{4}-12 i g t\left(A^{\dagger 2} A^{3}+3 A^{\dagger} A^{2}+2 A\right) B^{2} C-6 g^{2} t^{2} \\
& \times\left(A^{\dagger 2} A^{6}+3 A^{\dagger} A^{5}+2 A^{4}\right)\left(B^{\dagger 2} B^{2}+4 B^{\dagger} B+2\right) .
\end{aligned}
$$

The real quadrature component for fourth-order squeezing in fundamental mode is given as

$$
F_{1 A}(t)=\frac{1}{2}\left[A^{4}(t)+A^{\dagger 4}(t)\right]
$$

Using (23) and (48) in (49), we get the expectation values as

$$
\begin{aligned}
&\left\langle\psi\left|F_{1 A}(t)\right| \psi\right\rangle^{2}=\frac{1}{4}\left[\alpha^{8}+\alpha^{* 8}+2|\alpha|^{8}-24 g^{2} t^{2}\right. \\
& \times\left\{\left(|\alpha|^{4}+3|\alpha|^{2}+2\right)\left(\alpha^{8}+\alpha^{* 8}\right) \quad\right. \text { (50) } \\
&\left.\left.+2|\alpha|^{12}+6|\alpha|^{10}+4|\alpha|^{8}\right\}\right], \\
&\left\langle\psi\left|F_{1 A}^{2}(t)\right| \psi\right\rangle=\frac{1}{4}\left[\alpha^{8}+\alpha^{* 8}+2|\alpha|^{8}+16|\alpha|^{6}+72|\alpha|^{4}\right. \\
&+ 96|\alpha|^{2}+24-24 g^{2} t^{2} \\
& \times\left\{\left(|\alpha|^{4}+7|\alpha|^{2}+14\right)\left(\alpha^{8}+\alpha^{* 8}\right)\right. \\
&\left.\left.+2|\alpha|^{12}+18|\alpha|^{10}+64|\alpha|^{8}+68|\alpha|^{6}\right\}\right] .
\end{aligned}
$$

Therefore, subtracting (50) from (51), we obtain

$$
\begin{gathered}
{\left[\Delta F_{1 A}(t)\right]^{2}=\frac{1}{4}\left[16|\alpha|^{6}+72|\alpha|^{4}+96|\alpha|^{2}+24-24 g^{2} t^{2}\right.} \\
\times\left\{\left(4|\alpha|^{2}+12\right)\left(\alpha^{8}+\alpha^{* 8}\right)+12|\alpha|^{10}\right. \\
\left.\left.+60|\alpha|^{8}+68|\alpha|^{6}\right\}\right] .
\end{gathered}
$$

Using (23) and (36), we have

$$
\begin{aligned}
\frac{1}{4}\left\langle 16 N_{1 A}^{3}(t)+24 N_{1 A}^{2}(t)+56 N_{A}+24\right\rangle \\
=\frac{1}{4}\left[16|\alpha|^{6}+72|\alpha|^{4}+96|\alpha|^{2}+24\right. \\
\left.\quad-24 g^{2} t^{2}\left(12|\alpha|^{10}+60|\alpha|^{8}+68|\alpha|^{6}\right)\right] .
\end{aligned}
$$

Subtracting (53) from (52), we get

$$
\begin{aligned}
{\left[\Delta F_{1 A}(t)\right]^{2} } & -\frac{1}{4}\left\langle 16 N_{1 A}^{3}(t)+24 N_{1 A}^{2}(t)+56 N_{A}+24\right\rangle \\
= & -48 g^{2} t^{2}\left(|\alpha|^{10}+3|\alpha|^{8}\right) \cos 8 \theta .
\end{aligned}
$$

Using (28), we obtain the stimulated process as

$$
\begin{aligned}
& {\left[\Delta F_{1 A}(t)\right]^{2}-\frac{1}{4}\left\langle 16 N_{1 A}^{3}+24 N_{1 A}^{2}(t)+56 N_{1 A}(t)+24\right\rangle} \\
& =-24 g^{2} t^{2}\left(|\alpha|^{10}+3|\alpha|^{8}\right)\left(|\beta|^{4}+4|\beta|^{2}+2\right) \cos 8 \theta .
\end{aligned}
$$

The right-hand sides of (54) and (55) are negative, for all values of $\theta$ for which $\cos 8 \theta>0$, indicating the existence of squeezing in fourth-order field amplitude in the fundamental mode in the spontaneous and stimulated processes, respectively.

Using (23) and (36), the statistics of fundamental mode in six-wave mixing is found to be sub-Poissonian, given as

$$
\left\langle\Delta N_{1 A}\right\rangle^{2}-\left\langle N_{1 A}\right\rangle=-12 g^{2} t^{2}|\alpha|^{6} .
$$




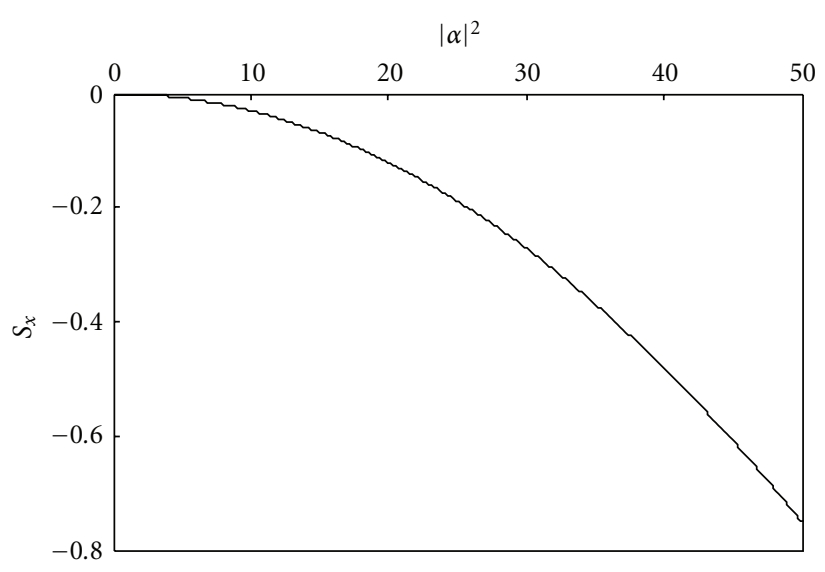

(a)

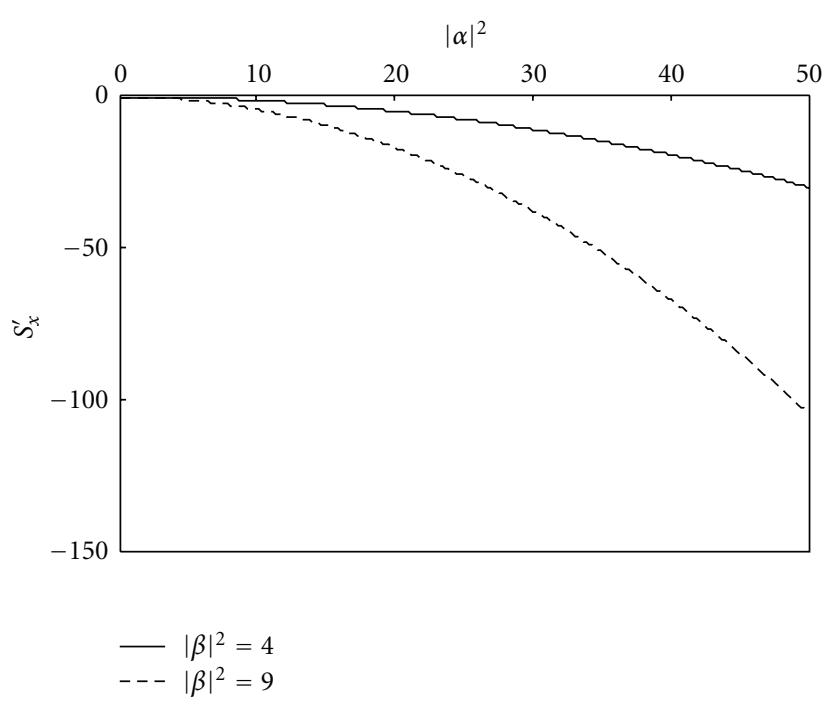

(b)

FIgURE 2: Dependence of first-order squeezing (a) $s_{X}$ with $|\alpha|^{2}$ in spontaneous and (b) $s_{X}^{\prime}$ with $|\alpha|^{2}$ and $|\beta|^{2}$ in stimulated six-wave mixing process.

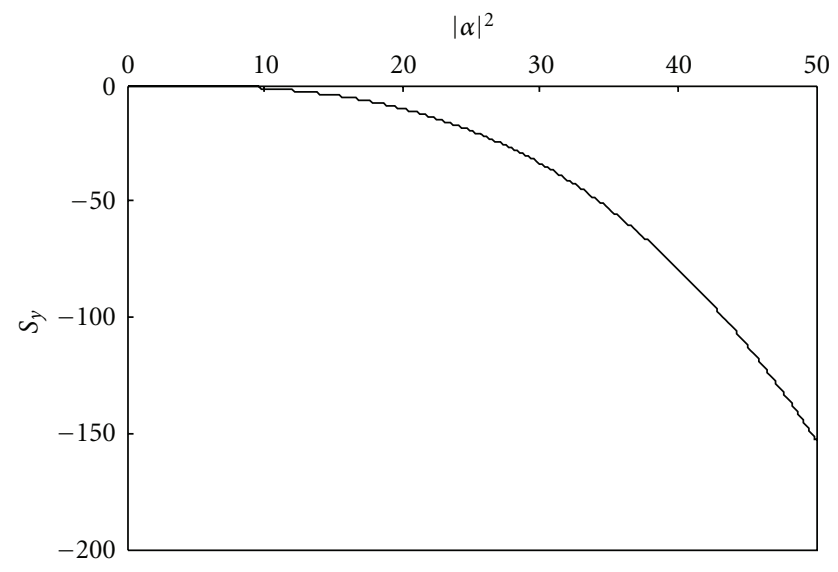

(a)

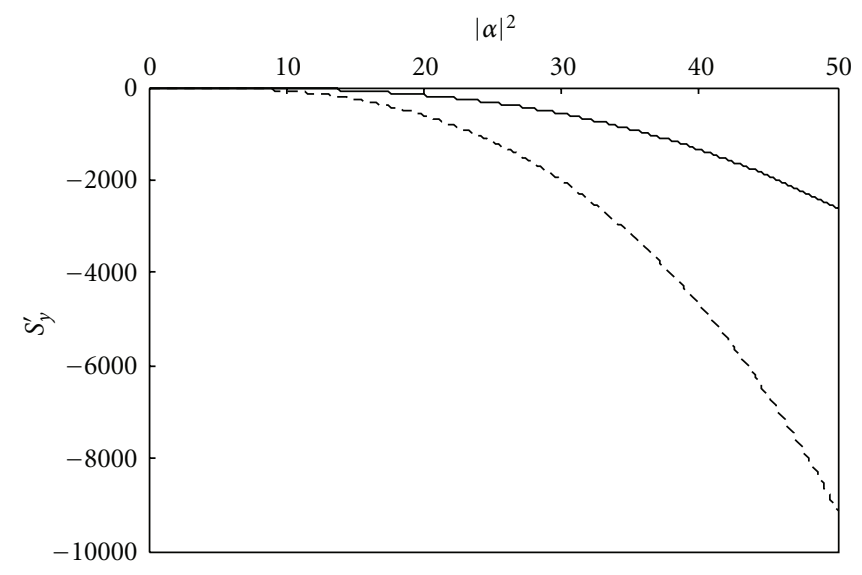

$$
\begin{aligned}
-|\beta|^{2} & =4 \\
---|\beta|^{2} & =9
\end{aligned}
$$

(b)

Figure 3: Dependence of amplitude-squared squeezing (a) $s_{y}$ with $|\alpha|^{2}$ in spontaneous and (b) $s_{y}^{\prime}$ with $|\alpha|^{2}$ and $|\beta|^{2}$ in stimulated six-wave mixing process.

\section{Signal-to-Noise Ratio}

Signal-to-noise ratio is defined as ratio of the magnitude of the signal to the magnitude of the noise. With the approximations $\theta=0$ and $|g t|^{2} \ll 1$, the maximum signal-to-noise ratio (in decibels) in field amplitude and higher orders is given in the following.

Using (25) and (26), signal-to-noise ratio in field amplitude is defined as

$$
\mathrm{SNR}_{1}=20 * \log _{10} \frac{\left\langle X_{1 A}(t)\right\rangle^{2}}{\left[\Delta X_{1 A}(t)\right]^{2}}=20 * \log _{10}\left(2|\alpha|^{2}\right) .
$$

Using (33) and (35), SNR in amplitude-squared squeezing is given as

$$
\mathrm{SNR}_{2}=20 * \log _{10} \frac{\left(2|\alpha|^{4}+|\alpha|^{2}\right)}{\left(3|\alpha|^{2}+2\right)} .
$$

Using (42) and (44), SNR in amplitude-cubed squeezing is expressed as

$$
\mathrm{SNR}_{3}=20 * \log _{10} \frac{\left(3|\alpha|^{4}+6|\alpha|^{2}+2\right)}{\left(9|\alpha|^{2}+18\right)} .
$$




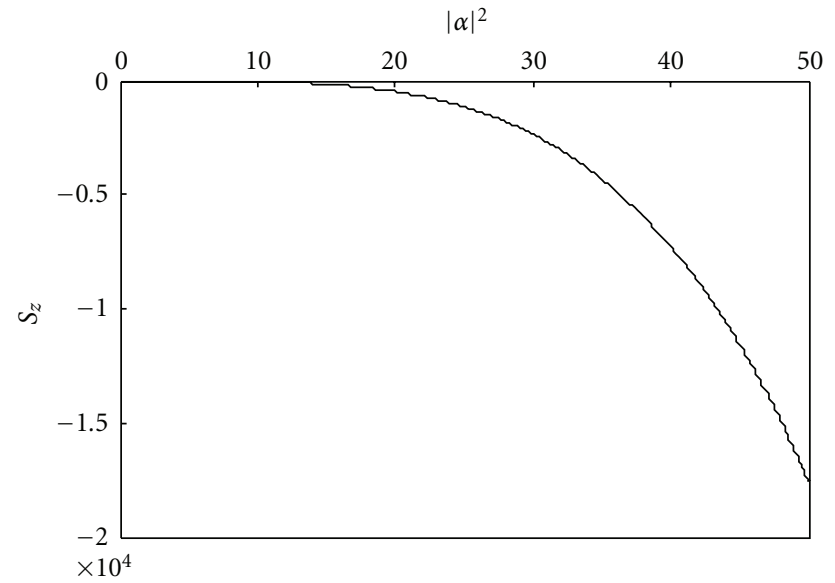

(a)

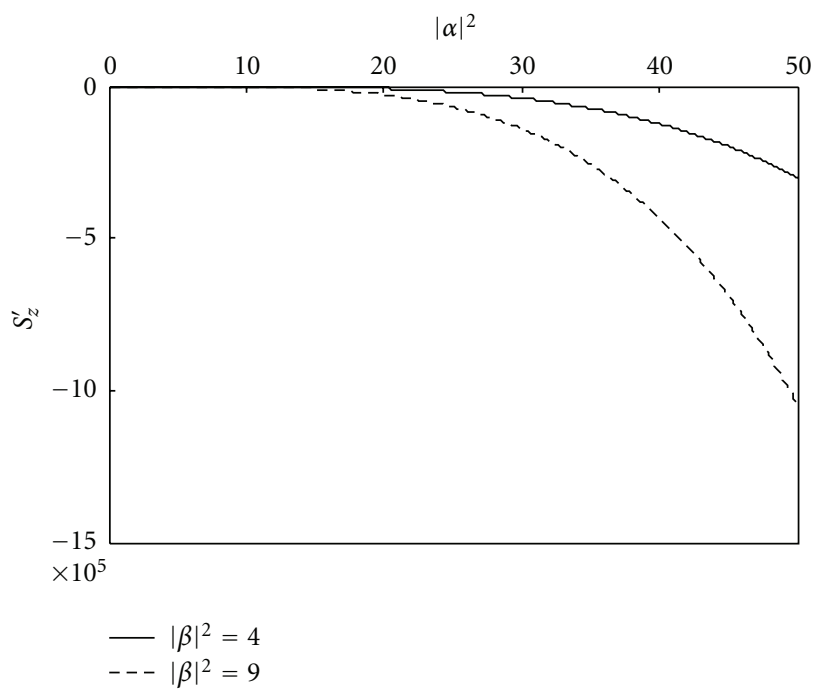

(b)

FIGURE 4: Dependence of amplitude-cubed squeezing (a) $S_{z}$ with $|\alpha|^{2}$ in spontaneous and (b) $S_{z}^{\prime}$ with $|\alpha|^{2}$ and $|\beta|^{2}$ in stimulated sixwave mixing process.

Using (50) and (52), SNR in fourth-order squeezing is expressed as

$$
\mathrm{SNR}_{4}=20 * \log _{10} \frac{\left(|\alpha|^{6}+3|\alpha|^{4}+2|\alpha|^{2}\right)}{\left(5|\alpha|^{4}+21|\alpha|^{2}+17\right)}
$$

\section{Results}

The results show the presence of squeezing in field amplitude, amplitude-squared, amplitude-cubed, and fourthorder field amplitude of fundamental mode in six-wave mixing process. To study squeezing, we denote the right-hand sides of relations (27), (38), (46), and (54) by $S_{x}, S_{y}, S_{z}$, and $S_{f}$ for spontaneous and right-hand sides of relations (30), (39), (47), and (55) by $S_{x}^{\prime}, S_{y}^{\prime}, S_{z}^{\prime}$, and $S_{f}^{\prime}$ for stimulated interaction for field amplitude, amplitude-squared, amplitude-cubed,

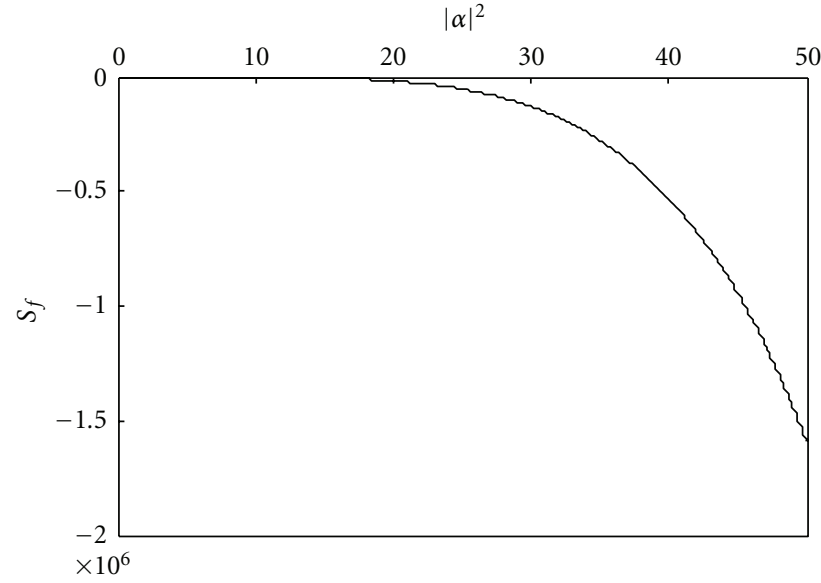

(a)

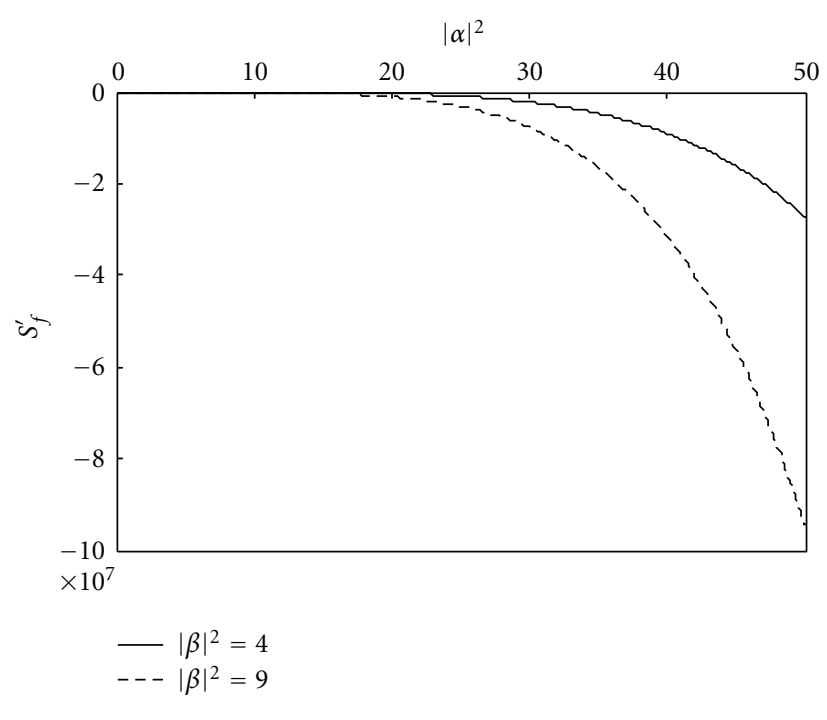

(b)

Figure 5: Dependence of fourth-order field amplitude squeezing (a) $S_{f}$ with $|\alpha|^{2}$ in spontaneous and (b) $S_{f}^{\prime}$ with $|\alpha|^{2}$ and $|\beta|^{2}$ in stimulated six-wave mixing process.

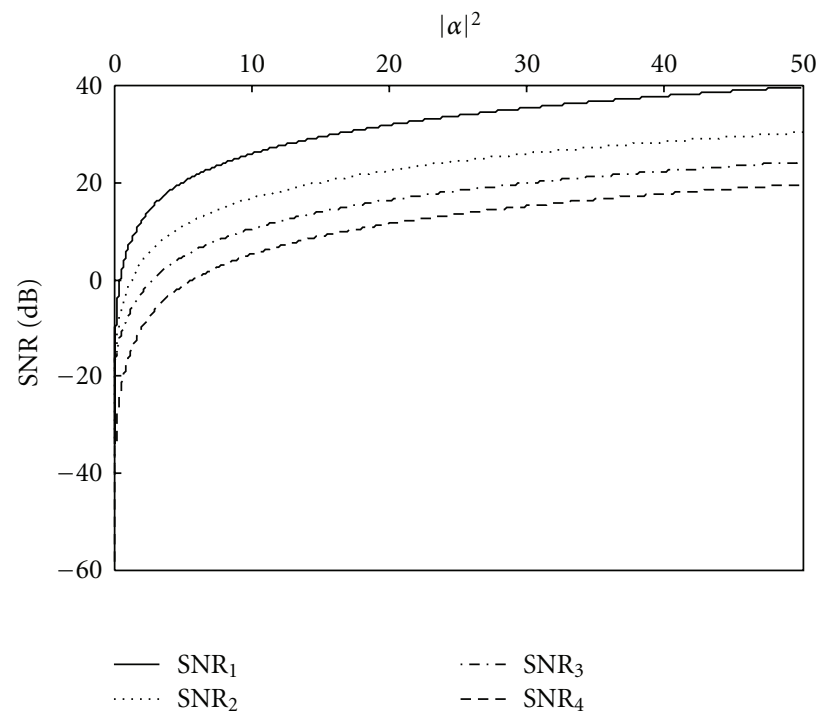

FIGURE 6: Signal-to-noise ratio for different order squeezing. 
and for fourth-order field amplitude, respectively. Taking $|g t|^{2}=10^{-4}$ and $\theta=0$, the variations of $S_{x}, S_{y}, S_{z}$, and $S_{f}$ with photon number $|\alpha|^{2}$ for spontaneous interaction and of $S_{x}^{\prime}, S_{y}^{\prime}, S_{z}^{\prime}$, and $S_{f}^{\prime}$ with $|\alpha|^{2}$ and $|\beta|^{2}$ for stimulated interaction are shown from Figures $2,3,4$, and 5.

A comparison between results of spontaneous and stimulated processes shows the occurrence of multiplication factor $\left(|\beta|^{4}+4|\beta|^{2}+2\right)$. It implies that squeezing in the fundamental mode in stimulated interaction is greater than corresponding squeezing in spontaneous interaction. It is also seen that maximum squeezing occurs when $\theta=0$. The signal-to-noise ratio is found to be higher in lower orders as shown in Figure 6.

\section{Conclusion}

Figures 2, 3, 4, and 5 show that squeezing increases nonlinearly with $|\alpha|^{2}$, which is directly dependent upon the number of photons. The squeezing in any order during stimulated interaction (Figures 5(b), 4(b), 3(b), and 2(b)) is higher than the squeezing in corresponding order in spontaneous (Figures $5,4,3$, and 2 ) interaction by a factor $\left(|\beta|^{4}+4|\beta|^{2}+2\right.$ ). The squeezing is higher in higher orders in both processes. Thus, the higher-order squeezing associated with higher order nonlinear optical processes makes it possible to achieve significant noise reduction.

It has also been found that the fundamental mode of field amplitude shows sub-Poissonian behavior as shown in relation (56). The signal-to-noise ratio is higher in lower orders squeezed states as reported earlier for Raman process [34].

\section{References}

[1] L. Mandel, "Squeezing and photon antibunching in harmonic generation," Optics Communications, vol. 42, no. 6, pp. 437439, 1982.

[2] M. Hillery, "Squeezing of the square of the field amplitude in second harmonic generation," Optics Communications, vol. 62, no. 2, pp. 135-138, 1987.

[3] C. K. Hong and L. Mandel, "Generation of higher-order squeezing of quantum electromagnetic fields," Physical Review A, vol. 32, no. 2, pp. 974-982, 1985.

[4] C. K. Hong and L. Mandel, "Higher-order squeezing of a quantum field," Physical Review Letters, vol. 54, no. 4, pp. 323325, 1985.

[5] Y. B. Zhan, "Amplitude-cubed squeezing in harmonic generations," Physics Letters A, vol. 160, no. 6, pp. 498-502, 1991.

[6] J. Lal and R. M. P. Jaiswal, "Amplitude-cubed squeezing in Kth harmonic generation," Indian Journal of Pure and Applied Physics, vol. 36, no. 9, pp. 481-484, 1998.

[7] C. M. Caves, "Quantum-mechanical noise in an interferometer," Physical Review D, vol. 23, no. 8, pp. 1693-1708, 1981.

[8] A. Buonanno and Y. Chen, "Improving the sensitivity to gravitational-wave sources by modifying the input-output optics of advanced interferometers," Physical Review D, vol. 69, no. 10, Article ID 102004, 29 pages, 2004.

[9] K. McKenzie, B. C. Buchler, D. A. Shaddock, P. K. Lam, and D. E. McClelland, "Analysis of a sub-shot-noise power recycled Michelson interferometer," Classical and Quantum Gravity, vol. 21, no. 5, pp. S1037-S1043, 2004.
[10] K. Goda, O. Miyakawa, E. E. Mikhailov et al., "A quantumenhanced prototype gravitational-wave detector," Nature Physics, vol. 4, no. 6, pp. 472-476, 2008.

[11] H. P. Yuen and J. H. Shapiro, "Optical communication with two photon coherent states-part I: quantum state propagation and quantum noise," IEEE Transactions on Information Theory, vol. 24, no. 6, pp. 657-668, 1978.

[12] N. Treps, N. Grosse, W. P. Bowen et al., "Nano-displacement measurements using spatially multimode squeezed light," Journal of Optics B: Quantum and Semiclassical Optics, vol. 6, no. 8, pp. S664-S674, 2004.

[13] M. T. L. Hsu, V. Delaubert, W. P. Bowen, C. Fabre, H. A. Bachor, and P. K. Lam, "A quantum study of multibit phase coding for optical storage," IEEE Journal of Quantum Electronics, vol. 42, no. 10, pp. 1001-1007, 2006.

[14] T. Eberle, S. Steinlechner, J. Bauchrowitz et al., "Quantum enhancement of the zero-area sagnac interferometer topology for gravitational wave detection," Physical Review Letters, vol. 104, no. 25, Article ID 251102, 4 pages, 2010.

[15] M. Mehmet, T. Eberle, S. Steinlechner, H. Vahlbruch, and R. Schnabel, "Demonstration of a quantum-enhanced fiber Sagnac interferometer," Optics Letters, vol. 35, no. 10, pp. 1665-1667, 2010.

[16] J. Peřina, V. Peřinová, C. Sibilia, and M. Bertolotti, "Quantum statistics of four-wave mixing," Optics Communications, vol. 49, no. 4, pp. 285-289, 1984.

[17] R. Loudon, "Squeezing in two-photon absorption," Optics Communications, vol. 49, no. 1, pp. 67-70, 1984.

[18] R. E. Slusher, L. W. Hollberg, B. Yurke, J. C. Mertz, and J. F. Valley, "Observation of squeezed states generated by four-wave mixing in an optical cavity," Physical Review Letters, vol. 55, no. 22, pp. 2409-2412, 1985.

[19] D. K. Giri and P. S. Gupta, "Short-time squeezing effects in spontaneous and stimulated six-wave mixing process," Optics Communications, vol. 221, no. 1-3, pp. 135-143, 2003.

[20] D. K. Giri and P. S. Gupta, "The squeezing of radiation in four-wave mixing processes," Journal of Optics B: Quantum and Semiclassical Optics, vol. 6, no. 1, pp. 91-96, 2004.

[21] S. Rani, J. Lal, and N. Singh, "Squeezing and photon statistical effects in spontaneous and stimulated eight-wave mixing process," Optical and Quantum Electronics, vol. 39, no. 2, pp. 157-167, 2007.

[22] G. S. Kanter and P. Kumar, "Enhancement of bright squeezing in the second harmonic by internally seeding the $\chi(2)$ interaction," IEEE Journal of Quantum Electronics, vol. 36, no. 8, pp. 916-922, 2000.

[23] A. Sizmann, R. J. Horowicz, G. Wagner, and G. Leuchs, "Observation of amplitude squeezing of the up-converted mode in second harmonic generation," Optics Communications, vol. 80, no. 2, pp. 138-142, 1990.

[24] S. T. Gevorkyan, G. Y. Kryuchkyan, and K. V. Kheruntsyan, "Noise, instability and squeezing in third harmonic generation," Optics Communications, vol. 134, no. 1-6, pp. 440-446, 1997.

[25] J. Lal and R. M. P. Jaiswal, "Amplitude squared squeezing and photon statistics in second and third harmonic generations," Indian Journal of Physics, vol. 72, pp. 637-642, 1998.

[26] J. Lal and R. M. P. Jaiswal, "Generation of amplitude-squared squeezed states by combination of up and down degenerate parametric processes," Indian Journal of Pure and Applied Physics, vol. 36, no. 8, pp. 415-418, 1998. 
[27] A. Kumar and P. S. Gupta, "Short-time squeezing in spontaneous Raman and stimulated Raman scattering," Quantum and Semiclassical Optics, vol. 7, no. 5, article 5, pp. 835-841, 1995.

[28] A. Kumar and P. S. Gupta, "Higher-order amplitude squeezing in hyper-Raman scattering under short-time approximation," Journal of Optics B: Quantum and Semiclassical Optics, vol. 8, no. 5, pp. 1053-1060, 1996.

[29] K. Kim, "Higher order sub-Poissonian," Physics Letters, Section A, vol. 245, no. 1-2, pp. 40-42, 1998.

[30] D. Erenso, R. Vyas, and S. Singh, "Higher-order subPoissonian photon statistics in terms of factorial moments," Journal of the Optical Society of America B, vol. 19, no. 6, pp. 1471-1475, 2002.

[31] H. Prakash and D. K. Mishra, "Higher order sub-Poissonian photon statistics and their use in detection of Hong and Mandel squeezing and amplitude-squared squeezing," Journal of Physics B: Atomic, Molecular and Optical Physics, vol. 39, no. 9, article 14, pp. 2291-2297, 2006.

[32] H. Prakash and P. Kumar, "Equivalence of second-order subPoissonian statistics and fourth-order squeezing for intense light," Journal of Optics B: Quantum and Semiclassical Optics, vol. 7, no. 12, pp. S786-S788, 2005.

[33] H. Prakesh and D. K. Mishra, "Corrigendum to Higher order sub-Poissonian photon statistics and their use in detection of Hong and Mandel squeezing and amplitude-squared squeezing," Journal of Physics B: Atomic, Molecular and Optical Physics, vol. 40, no. 12, article C01, pp. 2531-2532, 2007.

[34] D. K. Giri and P. S. Gupta, "Higher-order squeezing of the electromagnetic field in spontaneous and stimulated Raman processes," Journal of Modern Optics, vol. 52, no. 12, pp. 1769$1781,2005$. 

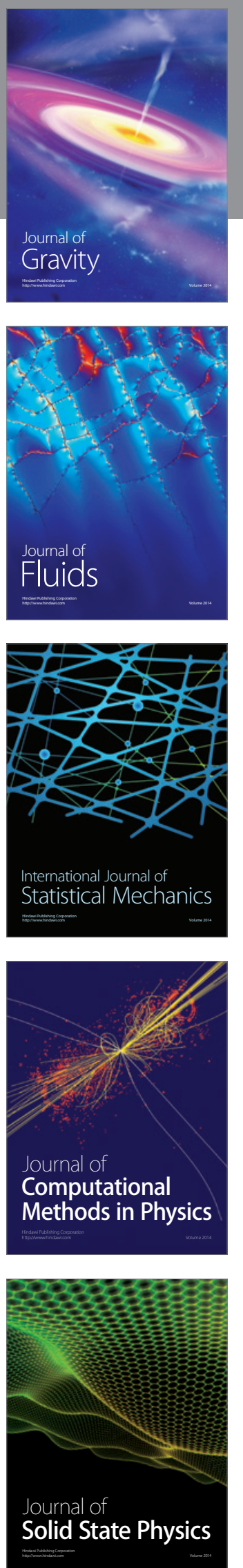



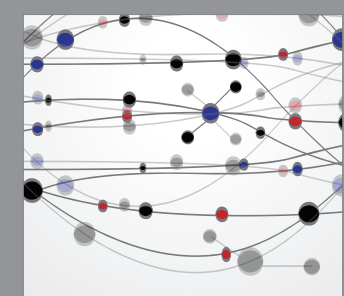

The Scientific World Journal
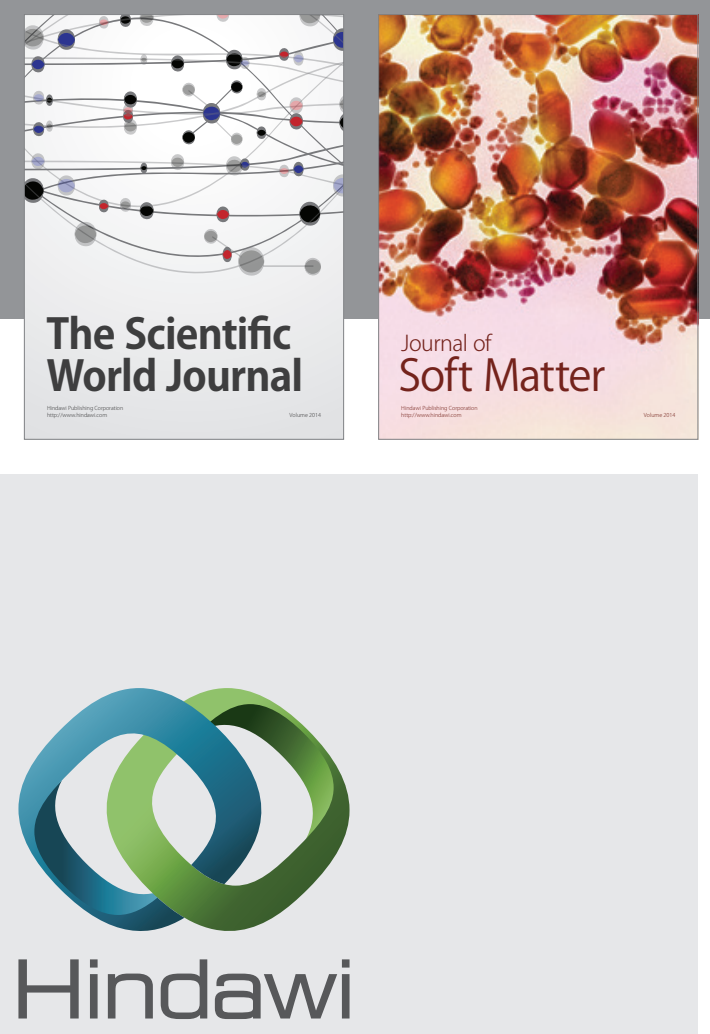

Submit your manuscripts at

http://www.hindawi.com
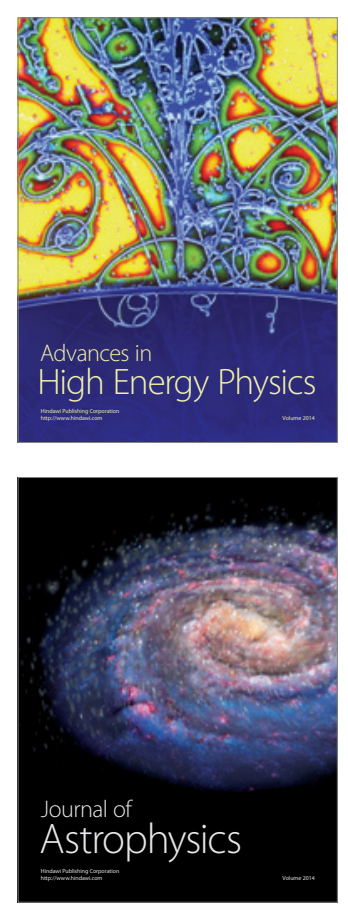
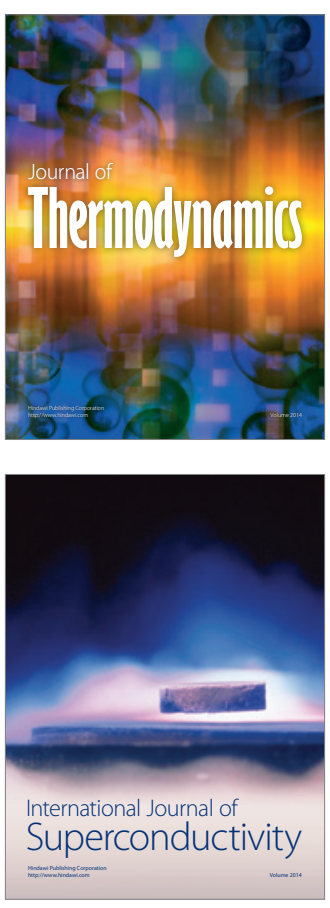
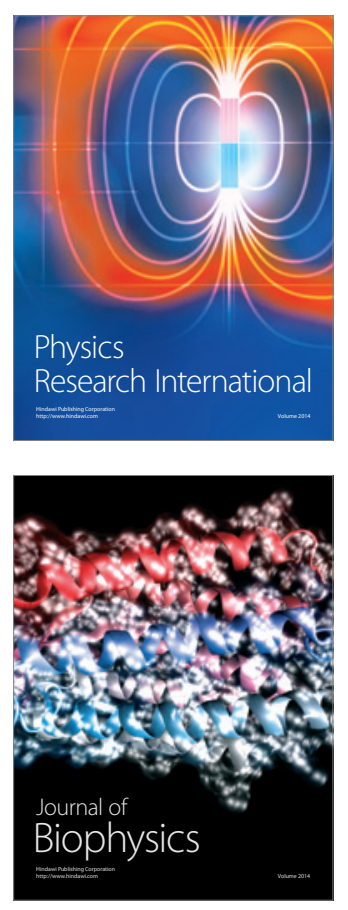
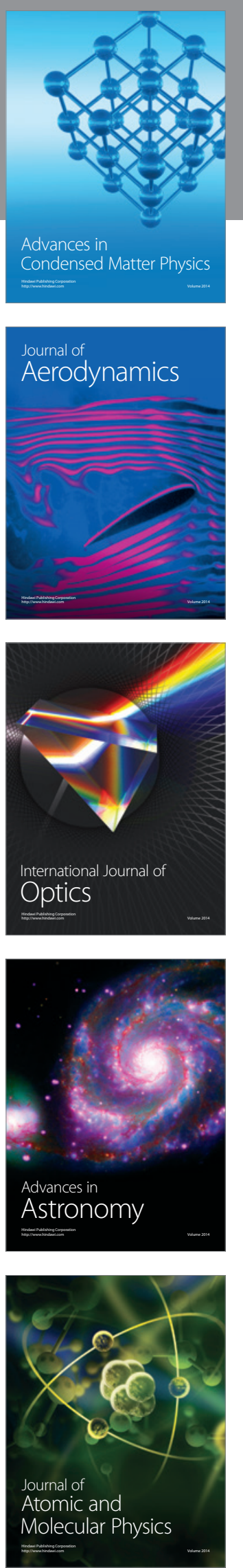